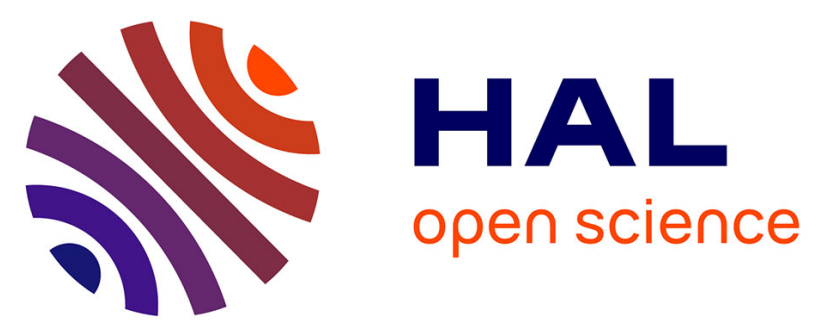

\title{
Application of BSIF, Log-Gabor and mRMR Transforms for Iris and Palmprint based Bi-modal Identification System
}

Bilal Attallah, Amina Serir, Youssef Chahir, Abdelwahhab Boudjelal

\section{- To cite this version:}

Bilal Attallah, Amina Serir, Youssef Chahir, Abdelwahhab Boudjelal. Application of BSIF, Log-Gabor and mRMR Transforms for Iris and Palmprint based Bi-modal Identification System. IEEE/International Conference on Advanced Technologies for Signal and Image Processing (ATSIP'2017), 2017, Fez, Morocco. 10.1109/ATSIP.2017.8075600 · hal-01621054

\section{HAL Id: hal-01621054}

\section{https://hal-normandie-univ.archives-ouvertes.fr/hal-01621054}

Submitted on 18 Sep 2018

HAL is a multi-disciplinary open access archive for the deposit and dissemination of scientific research documents, whether they are published or not. The documents may come from teaching and research institutions in France or abroad, or from public or private research centers.
L'archive ouverte pluridisciplinaire HAL, est destinée au dépôt et à la diffusion de documents scientifiques de niveau recherche, publiés ou non, émanant des établissements d'enseignement et de recherche français ou étrangers, des laboratoires publics ou privés. 


\title{
Application of BSIF, Log-Gabor and mRMR Transforms for Iris and Palmprint based Bi-modal Identification System
}

\author{
Bilal Attallah ${ }^{(1,2)}$,Amina Serir ${ }^{(2)}$, Youssef Chahir ${ }^{(1)}$ and Abdelwahhab Boudjelal (1) \\ (1) Normandie University, UNICAEN, ENSICAEN, CNRS, France \\ *bilal.attallah@unicaen.fr \\ Youssef.Chahir@unicaen.fr \\ Abdelwahhab.Boudjelal@unicaen.fr \\ (2) USTHB, LTIR Laboratory, Electronic Department, Algiers \\ aserir@usthb.dz
}

\begin{abstract}
Verification of individual identity through the process of biometric identification involves comparison between an encoded value and a stored value of the biometric feature in question. The effectiveness of a multimodal user authentication system is greater, but so is its complexity. The system error rate is reduced by the fact that multiple biometric features are combined, thus solving the weakness of the single biometric. Performance of individual authentication through palm-print- and iris-based bimodal biometric system is proposed in the present study. To this end, Log-Gabor filter and BSIF (Binarised Statistical Image Feature) coefficients are employed to obtain the iris and palmprint traits, and subsequently selection of the features vector is conducted with mRMR (Minimum Redundancy Maximum Relevance) transforms in higher coefficients. To match the iris or palm-print feature vector, the Hamming Distance is applied. According to the experiment outcomes, the proposed system not only has a significantly high recognition rate but it also affords greater security compared to the single biometric system.
\end{abstract}

Keywords-Biometric; Palm-print; Iris; Log-Gabor filter; BSIF; mRMR; Feature Fusion.

\section{INTRODUCTION}

Biometric technologies use one or multiple physical or behavioural features particular to an individual to automatically identify that individual. Basically, a biometric system is a system of automatic pattern identification that verifies certain features of an individual of interest with the purpose of confirming the identity of that individual. However, several limitations are presented by single biometric systems, including noise in the sensed biometric data, non-universality, and the fact that the selected biometric feature is non-distinct, resulting in significantly high error rates in person identification [1-3]. Multiple biometric systems are not affected by such limitations, their reliability being greater owing to the use of multiple templates security. Each multi-biometric system is created according to its intended usage. Many researchers have put forth various designs for such systems, which are differentiated by structure, the number and types of biometric modalities, as well as the techniques employed to integrate or combine data. A compact multi-biometric authentication system is presented in the current study, involving person identification based on the biometric modalities of iris and palm-print. A one-dimensional Log-Gabor filter is used to generate the feature vectors for the two modalities, which are then contrasted against enrolment templates separately stored for every biometric feature. Matching score computation is undertaken independently by every simple biometric system on the basis of the aspects that the feature vector and template have in common. Biometric fusion [4-5] is a way of integrating the individual modalities when more than one biometric feature is employed in an identification system. This study applies matching-score level fusion to merge the matching scores from the two authentication modalities into a single matching score. User validation or invalidation is decided according to this singular matching score.

The structure of the study's main body is as follows. Chapters 2 and 3 respectively focus on the framework of the suggested multi-biometric system and biometric modalities. The pre-processing procedure for iris and palm-print is explained in Chapter 4, while the procedures of feature extraction and encoding, alongside 1D Log-Gabor filter, BSIF and mRMR, are presented in Chapter 5. The similarity matching and the employed fusion approach are the focus of Chapter 6, while Chapter 7 discusses the performance assessment. Chapter 8 provides an overview and analysis of the experimental outcomes. Last but not least, Chapter 9 provides conclusions and recommendations for further research.

\section{PROPOSED SYSTEM}

The multi-biometric authentication system put forth in this study and involving palm-print and iris feature fusion is illustrated as a block-diagram in Figure 1. Image preprocessing, feature extraction and matching are the procedures of multi-biometric authentication for iris as well as palm-print modality. A singular feature is generated through the fusion of the feature scores from the two single biometric authentication systems and user validation or invalidation is decided according to this combined feature score. The benefits of each separate 
biometric are incorporated by this augmented structure, which can thus avoid the weaknesses of single biometric systems.

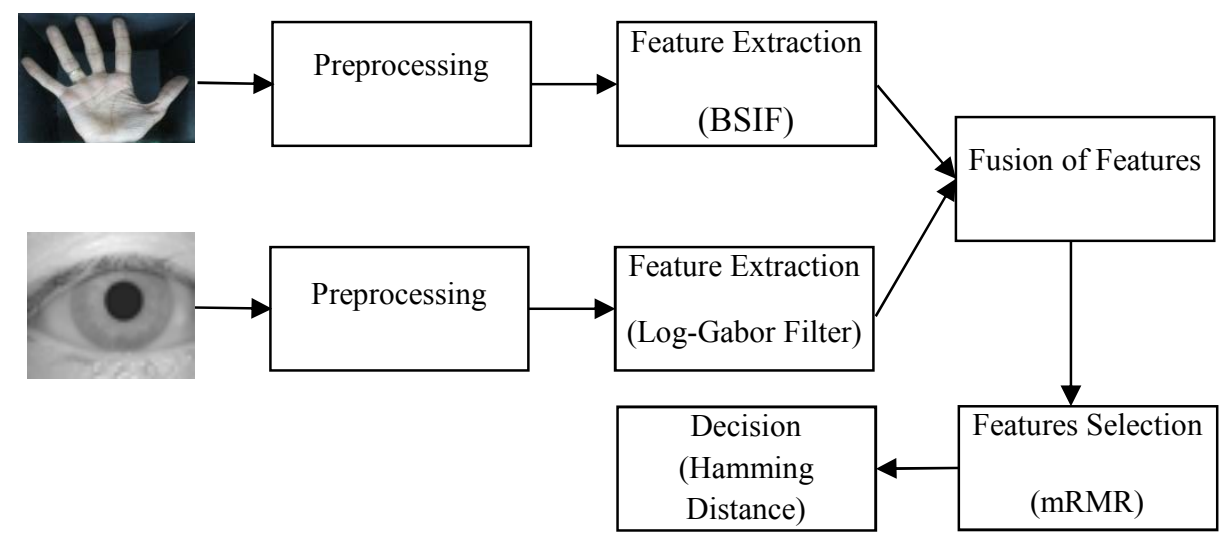

Fig. 1. Representation of the bimodal authentication system rooted in feature fusion in the form of a block-diagram

\section{BIOMETRIC MODALITIES}

\section{A. Iris recognition}

The circular ocular structure delimited by the pupil and sclera is known as the iris. It is considered that no two iris patterns are alike, which is why the iris has great potential as biometric feature [6]. Iris identification is deemed more useful than other biometric features to differentiate between individuals.

\section{B. Palmprint recognition}

The pattern on the internal side of the human hand is known as a palm-print and consists of several discriminating features, including main lines, wrinkles, ridges, minutiae points, and ture. What makes the palm-print a particularly useful biometric feature is the large palm surface from which traits can be extracted, ease of capture, and the fact that it is acceptable to most users. The palm-print is an obvious individual identifier [7], since each palm-print is unique and most people have no problem with having their palm-print processed. Hence, palmprint identification has potential for the development of moderately secure access control systems [8].

\section{PREPROCESSING PROCESS}

An important step in multi-biometric authentication systems that precedes feature extraction is image pre-processing. This procedure involves preparation of the obtained visual data (i.e. iris or palm-print) for extraction of features.

\section{A. Iris Preprocessing}

The processing of eye images is necessary to permit extraction of the singular iris traits for purposes of comparison. Pre-processing involves isolating the iris area in a digital eye image [9]. This area can be estimated with one circle for the iris/sclera boundary and another circle for the iris/pupil boundary. Furthermore, the iris area is covered on its superior side by the eyelid and on its inferior side by the eyelash line.

1) Segmentation: Localisation of the iris area is the next step following delineation of the external and internal circle boundaries (Figure 2). The boundary search is performed with the circular Hough Transform. Furthermore, the linear Hough Transform is employed to delimit the eyelids by applying two lines, while a simple threshold method is used for eyelash isolation [10].

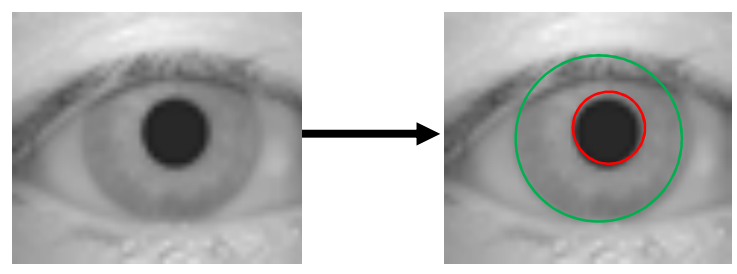

Fig. 2. Representation of an eye alongside iris segmentation

2) Normalization: Alignment of the segmented iris area to a fixed size is necessary to be able to compare irises. Daugman's rubber sheet model [11] is applied to undertake normalisation, involving mapping of the circular area to a rectangular. The pupil centre serves as the reference point during normalisation and the radial vectors circumscribe the iris area. The product of encoding is a bitwise template with several bits of information and an equivalent noise mask associated with the corrupt portions of the iris pattern. Template bits are correspondingly marked as corrupt. An iris 
with boundaries, normalisation and the noise mask are illustrated in Figure 3.

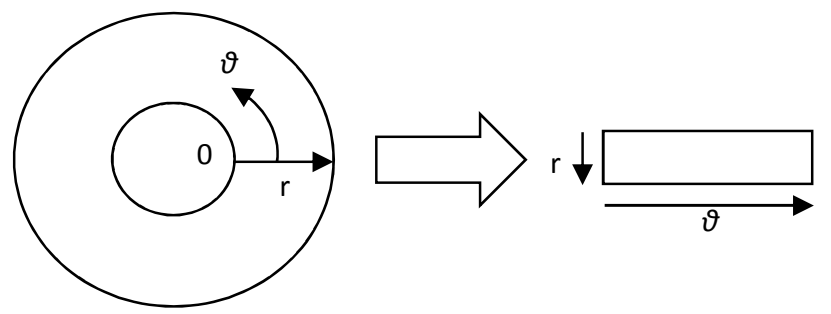

Fig. 3. Daugman's rubber sheet model

\section{B. Palmprint Preprocessing}

Palm image pre-processing must be conducted before the palm area can be localised. In this study, palm-print alignment is done with the pre-processing method proposed in [12], which involves image smoothing with the Gaussian smoothing filter, followed by extraction of the Region of Interest (ROI) and its traits. Hand binarisation is subsequently performed with Otsu's thresholding. The hand contour is obtained with the help of a contour-following algorithm. The next step is computation of the tangent of the two fixed points on the hand contour (i.e. the point between forefinger and middle finger and the point between ring finger and little finger), followed by palm-print alignment. Cropping of the middle portion of the image $(128 \times 128)$ is done so that it represents the entire palm-print. A mask is created to determine where the non-palm-print pixels are located and to eliminate image information that is irrelevant. A captured palm-print, ROI sub-image and associated mask are depicted in Figure 4.

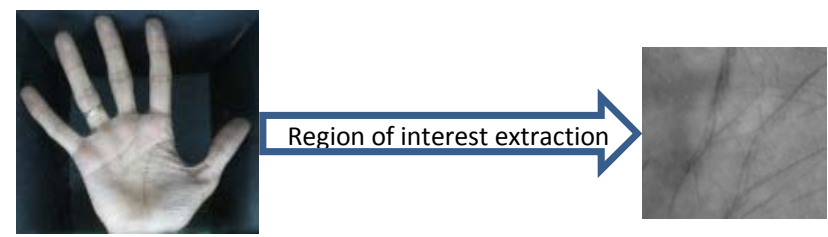

Fig. 4. Extraction of ROI

\section{FEATURE EXTRACTION, FUSION AND SELECTION}

Extraction of the data within iris and palm-print patterns with the highest degree of discrimination is essential. To ensure that templates can be compared, encoding should be restricted to the main traits of the iris and palm-print.

\section{A. Log-Gabor Filter}

Texture examination is usually conducted on the basis of Gabor features, as they effectively enable spatial and frequency data to be determined at the same time. However, the Gabor filter does have a limitation, namely, that a DC element is displayed by the symmetric filter when the bandwidth exceeds one octave [13]. This limitation can be addressed by employing the Log-Gabor filter type, which does not generate DC elements regardless of bandwidth because it is Gaussian on a logarithmic scale. The frequency response of this type of filter can be determined as follows:

$$
G(x, y)=\frac{1}{2 \pi \sigma^{2}} \exp \left[-\frac{\left(x^{2}+y^{2}\right)}{2 \sigma^{2}}\right] * \exp \left(i 2 \pi f_{c}\left(\sqrt{\left(x^{2}+y^{2}\right)}\right)\right.
$$

In the above, the central frequency of the filter is denoted by $\mathrm{f}$ and the scale deviation of the filter is denoted by $\sigma$. In general, these two parameters are selected in particular empirical work. Moreover, for every coordinate (x, y), eight potential orientations are provided by the Gabor filter.

\section{B. Binarized Statistical Image Features}

Kannala and colleagues were the first to propose BSIF [14], which is a technique that gives a binary code string for image pixels. The local descriptor of the image around a pixel is the code value of that pixel. Calculation of the filter response $R_{i}$ with an image $I_{p}$ and an equally sized linear filter $W_{i}$ takes the following form:

$$
\mathrm{R}_{\mathrm{i}}=\sum_{\mathrm{m}, \mathrm{n}} \mathrm{I}_{\mathrm{p}}(\mathrm{m}, \mathrm{n}) \mathrm{W}_{\mathrm{i}}(\mathrm{m}, \mathrm{n})
$$

In the above, the size of the PPI patch is indicated by $\mathrm{m}$ and $\mathrm{n}$, while $W_{i}$ represents the number of linear filters $\forall i=\{1,2, \ldots \ldots, n\}$ with responses that can be subjected to combined computation and binarisation to generate the binary string, as specified below [15]:

$$
b_{i}= \begin{cases}1 & \text { if } R_{i} \succ 0 \\ 0 & \text { otherwise }\end{cases}
$$

Representation of the BSIF codes takes the form of a histogram of pixel binary codes that are capable of differentiating the texture traits of the PPI. The suitability of the BSIF descriptor for palm-print authentication depends on the size of the filter and the bit string length.
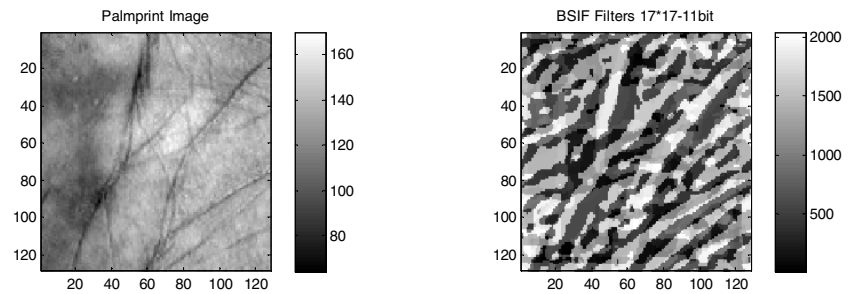

Fig. 5. Samples derived from the MSPolyU database and equivalent BSIF codes

\section{Feature Fusion}

Feature level fusion performs well and it is automatic and uncomplicated, which is why it is extensively employed [16]. This technique is applied in the present study to merge the outputs of the iris and palm-print feature modules. The final decision is formulated based on the single feature obtained from the integration of the two features. The fusion takes the following form: 


$$
\text { Fused }_{\text {vector }}=\left[e_{1}, e_{2}, e_{3}, \ldots, e_{n}, i_{1}, i_{2}, i_{3}, \ldots . i_{n}\right]
$$

In the above, the normalised feature vector of the Log Gabor filter is denoted by $E_{I}=\left[e_{1}, e_{2}, e_{3}, \ldots, e_{n}\right]$, while the normalised feature vector of BSIF is denoted by $I_{I}=\left[i_{1}, i_{2}, i_{3}\right.$, $\left.\ldots, i_{n}\right]$.

\section{Feature selection}

The correlation between the feature and target class enables the filter technique called Minimum Redundancy and Maximum Relevance (mRMR) to choose the features that are most relevant to the target class [17]. It represents an approximation for the purpose of ensuring that the joint distribution of the class label and the chosen features are as closely dependent as possible. Reciprocal information is employed by mRMR in a process to satisfy the optimisation criteria. If the subset of features and the number of features in that subset are respectively denoted by $\mathrm{S}$ and $|\mathrm{S}|$, then the minimal redundancy condition is calculated as:

$$
\min R(S), R(S)=\frac{1}{|S|^{2}} \sum_{x_{x, j}, \in S} I\left(x_{i}, x_{j}\right)
$$

The measure of relevance of a feature is given by the reciprocal information between that feature and the target class c. Based on this, the maximal relevance can be determined as:

$$
\max D(S, c), D(S, c)=\frac{1}{|S|} \sum_{x, S S} I\left(x_{j}, c\right)
$$

The conditions in equations (5) and (6) optimise the set of features chosen by mRMR at the same time. Feature ordering is used in this study, being ranked with the help of mRMR and according to certain measurements like redundancy and relevance. As indicated by the findings, feature ordering is both time-efficient and displays a greater classification performance than that reported in earlier research [17].

\section{MATCHING PROCESS}

When the Log Gabor filter and BSIF are applied, a template is generated that can be used to extract the features. Normalised Hamming Distance between the two representations permits the extracted features to be matched to the stored features, which in this case are the iris and palm-print.

If the chosen feature vectors are considered to be denoted by $U=\left(\lambda_{1}, \lambda_{2}, \ldots, \lambda_{10}\right)$ and the reference features are denoted by $Q=$ $\left(\rho_{1}, \rho_{2}, \ldots, \rho_{10}\right)$, then the HD can be determined in the following way:

$$
H D=\sqrt{\frac{1}{2} \sum_{i=1}^{B} \frac{\left(\lambda_{i}-\rho_{i}\right)^{2}}{\left(\lambda_{i}+\rho_{i}\right)}}
$$

\section{PERFORMANCE EVALUATION}

\section{A. Experimental database}

A database of palm-print and iris images is necessary for the performance assessment of the suggested multi-biometric identification system. Thus, the CASIA iris database [19] and the palm-print database of the Hong Kong Polytechnic University (PolyU) [20] have been consulted to create the multi-biometric database required for the experimental work in this study. For each of the 200 participants, twelve images (six iris and six palm-print images) are included in the multibiometric database.

\section{B. Results and discussion}

Multi-biometric fusion is intended to identify persons more accurately and more reliably compared than single biometric systems. A series of comparisons with single biometric systems (iris and palm-print), feature fusion, and feature selection are conducted to demonstrate the superior efficiency of the suggested system.

The ROC (Receiver Operating Characteristic) analysis facilitates assessment of biometric system performance based on the plotting of GAR (Genuine Acceptance Rate) against FAR (False Acceptance Rate). The GAR is expressed as GAR $=1$ FRR (False Reject Rate). The threshold that is associated with the maximal GAR equivalent to the minimal FAR must be chosen from the ROC curve. Results with minimum error and smallest code size (one dominant energy) are compared in Figure 5. In the case of the iris feature, the highest individual authentication rate $(\mathrm{GAR}=100 \%)$ is attained at $5.45 \% \mathrm{FAR}$, while in the case of the PolyU palm-print, this rate is achieved at $0.43 \%$ FAR. This confirms that the proposed algorithm is efficient for both databases.

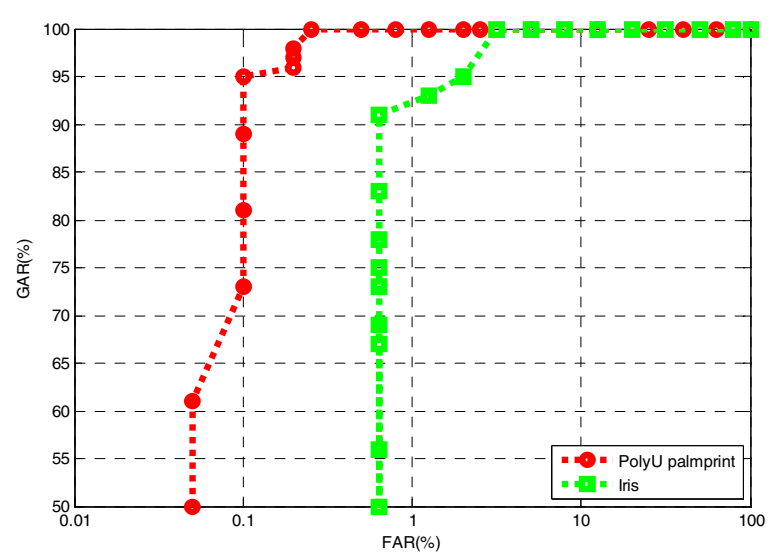

Fig. 6. ROCs associated with the iris and PolyU palm-print

Since a FAR of just $0.56 \%$ is associated with $100 \%$ GAR, the results obtained through the feature fusion of the iris and PolyU palm-print are more reliable compared to those obtained independently with the two features (Figure 6).

If a zero value is obtained for HD, then the histograms of $U$ and $Q$ are similar, but not identical [18]. 


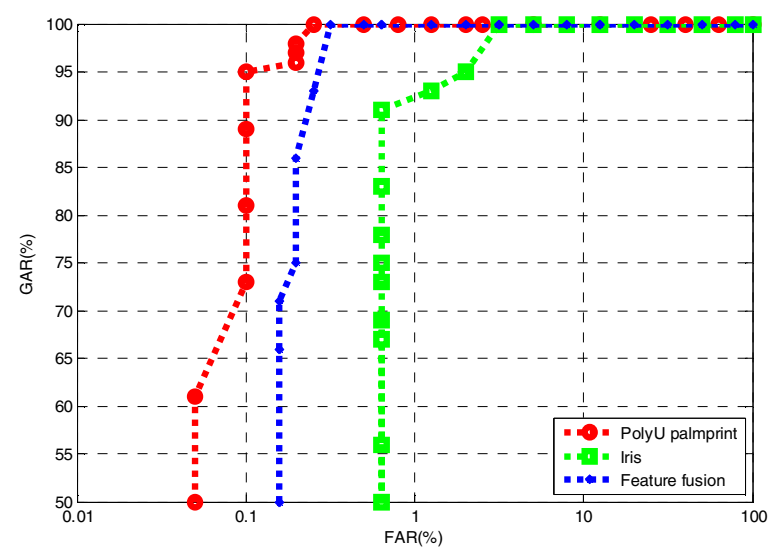

Fig. 7. ROCs associated with iris, PolyU palm-print and feature fusion

At a FAR of $0.23 \%$, a GAR of $100 \%$ is achieved with feature selection based on mRMR transforms (Figure 7).

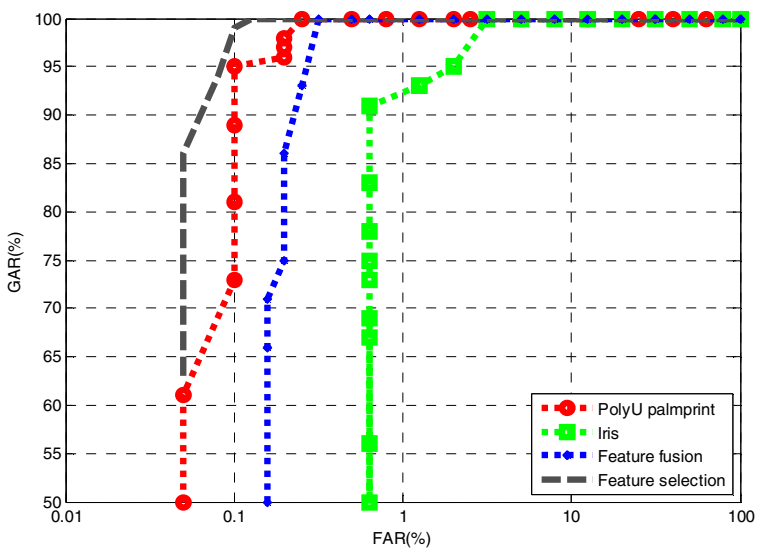

Fig. 8. ROCs associated with iris, PolyU palm-print, feature fusion and feature selection

Table 1 shows that, by comparison to other systems, the suggested system performs well.

TABLE I. PERFROMANCE OF THE SUGGESTED SYSTEM

\begin{tabular}{|c|c|c|c|c|}
\hline Ref no & Feature extraction & Level Fusion & $\begin{array}{c}\text { Template } \\
\text { size(Person) }\end{array}$ & $\begin{array}{c}E E R \\
(\%)\end{array}$ \\
\hline \multirow{4}{*}{$\begin{array}{c}\text { Proposed Scheme } \\
{[21]} \\
{[22]} \\
{[23]} \\
{[24]}\end{array}$} & Log Gabor+BSIF++mRMR & Feature fusion and Selection & 200 & 0.23 \\
\hline & Log Gabor & Score fusion & 100 & 0.21 \\
\hline & Block Truncation Coding & Score Fusion & 60 & 3.2 \\
\hline & 2D Gabor filter & Score fusion, Rank fusion & 173 & 5.3 \\
\hline
\end{tabular}

\section{CONCLUSION}

A biometric authentication system with combined iris and palm-print features has been proposed in the present study. Extraction of the differentiating features has been undertaken with the one-dimensional Log Gabor filter and BSIF. To make the system perform better, feature fusion has been applied. The system was assessed with the help of a database containing the information of 200 individuals. The outcome of the experiments revealed that the authentication process was improved by feature fusion information. The results achieved confirmed that the suggested system can be employed in high-security applications.

\section{REFERENCES}

[1] Fenghua wang, Jiuqiang han,"Robust Multimodal Biometric Authentication Integrating Iris, Face and almprint", information technology and control, vol.37, no.4, july 2008, pp.326-332.

[2] Kisku, A. Rattani, P. Gupta and J. Sing, "Biometric Sensor Image Fusion for Identity Verification: A Case Study with WaveletBased Fusion Rules Graph Matching," in Proc. of IEEE Conference on Technologies for Homeland Security, HST '09, Boston, pp. 433- 439, 11-12 May 2009.

[3] S.Hariprasath and T. Prabakar,"Multimodal Biometric Recognition using Iris Feature Extraction and Palmprint Features," in Proc of International Conference on Advances in Engineering, Science and Management (ICAESM), Nagapattinam, pp. 174-179,30-31 March 2012.

[4] A.Kumar, M. Hanmandu and S. Vasikarla, "Rank Level Integration of Face Based Biometrics," in Proc. of Ninth International Conference on Information Technology: New Generations (ITNG), Las Vegas, pp. 3641, 16-18 April 2012.

[5] A.Yazdanpanah, K. Faez and R. mirfattahi,"Multimodal Biometric System using Face, Ear and Gait Biometrics," in Proc. of 10th International Conference on Information Sciences Signal Processing and their Applications (ISSPA), Kuala Lumpur, pp. 251254, 10-13 May 2010.

[6] R. Prashanth, Shashikumar D.R., K. B. Raja, K. R. Venugopal, L. M. Patnaik,"High Security Human Recognition System using Iris Images", International Journal of Recent Trends in Engineering, Vol. 1, No. 1, May 2009, pp. 647-652.

[7] Wai kin kong and david zhang, Palmprint Texture Analysis On Low Resolution Images For Personal Authentication, IEEE Trans. on Pattern Analysis and Machine Intelligence, 2002.

[8] M. Wong, D. Zhang, W.K. Kong and G. Lu,', Real-time Palmprint Acquisition System Design', IEE Proc.-Vis. Image Signal Process. Vol.152, No. 5, October 2005.

[9] Kaushik Roy and Prabir Bhattacharya,"Optimal Features Subset Selection and Classification for Iris Recognition", Journal on Image and Video Processing, March 2008.

[10] XIE Mei, "Iris Recognition Technique", Journal of Electronic Science and Technology of China, Vol.4 No.3, Sep. 2006, pp. 219-224.

[11] J. G. Daugman, BThe importance of being random: Statistical principles of iris recognition,Pattern Recognition, vol. 36, pp. 279291, 2003.

[12] Zhang, W. Kong, J. You, and M. Wong, "On-line Palmprint Identification", IEEE Trans. on PAMI, vol. 25, no. 9, pp. 1041-1050.

[13] Peng Yao, Jun Li, Xueyi Ye, Zhenquan Zhuang and Bin Li, "Iris Recognition Algorithm Using Modified Log-Gabor Filters," 18th International Conference on Pattern Recognition (ICPR'06), Hong Kong, 2006, pp. 461-464.

[14] J. Kannala, E. Rahtu, Bsif: Binarized statistical image features, In Pattern Recognition (ICPR), 2012, pages 1363-1366. IEEE, 2012.

[15] Boukhari, A. Serir, "Weber Binarized Statistical Image Features (WBSIF) based video copy detection', Journal of Visual Communication and Image Representation 34 (2015) 50-64.

[16] Fenghua W, Jiuqiang H and Xianghua Y, 'Iris Recognition Based on Multialgorithmic Fusion", wseas transactions on information science \& applications, Volume 4, December 2007, pp. 1415-1421. 
[17] Peng H, Long F, Ding C. Feature Selection Based On Mutual Information: Criteria Of Max-Dependency, Max-Relevance, And Min-Redundancy. IEEE Trans Pattern Analysis And Machine Intelligence. 2005; 27(8), pp.1226-38.

[18] K. P. Hollingsworth, K. W. Bowyer and P. J. Flynn, "Improved Iris Recognition through Fusion of Hamming Distance and Fragile Bit Distance," in IEEE Transactions on Pattern Analysis and Machine Intelligence, vol. 33, no. 12, pp. 2465-2476, Dec. 2011.

[19] CASIA Iris Image Database, http://www.sinobiometrics.com.

[20] The Hong Kong Polytechnic University, PolyU Palmprint Database, http://www.comp.polyu.edu.hk/ biometrics.

[21] Kadri, A. Meraoumia, H. Bendjenna and S. Chitroub, "Palmprint \& iris for a multibiometric authentication scheme using Log-Gabor filter response," 2016 International Conference on Information Technology for Organizations Development (IT4OD), Fez, 2016, pp. 1-5.

[22] Ingwang L, Yan H and Jingyan W,' 'Fusing iris and palmprint at image level for multi-biometrics verification'. Proc. SPIE 8350, Fourth
International Conference on Machine Vision (ICMV 2011): Computer Vision and Image Analysis; Pattern Recognition and Basic Technologies, 83501Q (January 12, 2012)

[23] Manisha Madane, Sudeep Thepade, Score Level Fusion Based Bimodal Biometric Identification Using Thepade's Sorted n-ary Block Truncation Coding with Variod Proportions of Iris and Palmprint Traits, Procedia Computer Science, Volume 79, 2016, Pages 466-473, ISSN 1877-0509.

[24] Naderi, B. H. Soleimani, S. Matwin, B. N. Araabi and H. SoltanianZadeh, "Fusing Iris, Palmprint and Fingerprint in a Multi-biometric Recognition System," 2016 13th Conference on Computer and Robot Vision (CRV), Victoria, BC, 2016, pp. 327-334. 